\title{
(4HS) \\ Enhanced Emission Using Thin Li-Halide Cathodic Interlayers for Improved Injection into Poly(p-phenylene vinylene) Derivative PLEDs
}

\author{
Woo-Jun Yoon, ${ }^{a}$ Scott B. Orlove, ${ }^{\mathrm{a}, \mathrm{c}}$ Robert L. Olmon, ${ }^{\text {a,d }}$ and Paul R. Berger ${ }^{\mathrm{a}, \mathrm{b}, \mathrm{z}}$ \\ ${ }^{a}$ Department of Electrical and Computer Engineering, ${ }^{b}$ Department of Physics, The Ohio State University, \\ Columbus, Ohio 43210, USA
}

In this study, the effects of thin Li-halide cathodic interlayers on electron injection were examined for electroluminescent layers of polymer light-emitting diodes (PLEDs). An order of magnitude increase in current density is observed as Li-halide salts are varied down the group VII column of the periodic table. When considering luminance, devices with a $\mathrm{LiCl}$ interlayer were $2.3 \times$ greater than those with $\mathrm{LiF}$, whereas devices with $\mathrm{LiBr}$ were $2.8 \times$ greater, while concurrently lowering the turn-on voltage. This resulting enhanced current density and subsequent luminance could be due to a lowered work-function difference at the cathode created by either the Li-halides dissociation-induced doping of the polymer surface or an interfacial dipole of ionic Li-halide compound, leading to band bending.

(C) 2008 The Electrochemical Society. [DOI: 10.1149/1.2961823] All rights reserved.

Manuscript submitted June 2, 2008; revised manuscript received June 30, 2008. Published August 4, 2008

One of the important rate-limiting steps for high-efficiency polymer light-emitting diodes (PLEDs) is charge injection into the polymer semiconductor. It has been known that the interface, and subsequent potential barrier, between the active light-emitting conjugated polymer layer and the metal cathode plays an important role in controlling charge injection across this interface. ${ }^{1}$ Because most light-emitting conjugated polymers are good hole transporters but poor electron transporters, electron injection becomes a critical issue for balanced current components. It has been shown by numerous groups that the insertion of a thin compound interlayer, such as alkaline earth metal fluorides (i.e., $\mathrm{LiF}$ and $\mathrm{CsF}$ ), between the $\mathrm{Al}$ cathode and the electroluminescent polymer improves PLED device performance by enhancing electron injection. ${ }^{2-4}$ The physical mechanism behind this improved performance still remains a topic of some speculation ${ }^{5-7}$ However, two competing mechanisms have been proposed: a metal-halide dissociation introducing a localized surface doping effect, thus creating a low work-function contact at the interface and an interfacial dipole created by the polar metal halides, leading to strong localized band bending., ${ }^{8,9}$

Previous work has principally investigated fluoride-based chemistries, and this study extends that work by examining other halide-based chemistries ( $\mathrm{LiF}, \mathrm{LiCl}$, and $\mathrm{LiBr}$ ), which are considered salts. PLEDs using poly[2-methoxy-5-(2'-ethyl-hexyloxy)-1, 4-phenylenevinylene] (MEH-PPV) with various thin metal-halide interlayers between the electroluminescent layer and $\mathrm{Al}$ cathode were fabricated and tested. This study examined the effects of metal halides on the electron injection into the electroluminescent layers of PLEDs from their current density-voltage $(J-V)$ and luminancevoltage $(L-V)$ characteristics and electroluminescence (EL) emission spectra of the devices.

Patterning of the indium-tin-oxide (ITO) coatings was performed to define eight finger-shaped transparent anodes using conventional photolithography and $\mathrm{HCl}$-based wet etching. The ITO film thickness and sheet resistance were $\sim 1500 \AA$ and $10 \Omega \mathrm{cm}$, respectively. After photolithographic patterning, the anodes were plasma treated using an inductively coupled plasma reactive ion etching system with oxygen at a radio frequency power of $80 \mathrm{~W}$ to modify the surface work function. ${ }^{10}$ Poly(styrenesulfonate)-doped poly(3, 4-ethylenedioxythiophene) (PEDOT:PSS, Baytron P) layers were then added on top of the ITO anodes by spin coating, then annealed in an oven at $110^{\circ} \mathrm{C}$ for $10 \mathrm{~min}$ in ambient air. Thin films of the MEH-PPV were then spin-coated on top of the PEDOT:PSS layer

Present address: Northrop-Grumman Electronic Systems, Linthicum, Maryland 21090, USA

d Present address: Department of Electrical Engineering, University of Washington, Seattle, Washington 98195, USA.

E-mail: pberger@ieee.org from a $0.5 \%$ MEH-PPV solution in $80 \%$ toluene and $20 \%$ tetrahydrofuran. The conjugated polymers used in this study were purchased commercially and did not undergo any chemical modification or purification other than modest filtration.

Lithium-halide compounds $(>99.99 \%)$ for the interlayer between the EL layer and Al cathode were commercially obtained from Aldrich, Inc. Lithium-halide interlayers were evaporated thermally at pressures below $10^{-6}$ Torr with thicknesses nominally $5 \mathrm{~nm}$. The PLEDs were completed by shadowmask evaporation $\left(<\sim 10^{-6}\right.$ Torr $)$ of an $\mathrm{Al}$ cathode, about $100 \mathrm{~nm}$ thick. The final device structure is ITO/PEDOT:PSS/MEH-PPV/Li-halide/Al.

In order to investigate the role of thin $\mathrm{Li}$-halide interlayers between the MEH-PPV and $\mathrm{Al}$ on the device performance of PLEDs, control devices were fabricated and tested. First, a control device without Li-halides (ITO/PEDOT:PSS/MEH-PPV/Al) was tested. Next, control devices incorporating metallic Li (ITO/PEDOT:PSS/ $\mathrm{MEH}-\mathrm{PPV} / \mathrm{Li} / \mathrm{Al}$ ) were tested. All fabrication steps were performed in an inert glove box environment with $\leqslant 1 \mathrm{ppm}$ level of oxygen and water.

Highly conductive leads were attached to each patterned ITO anode surface along an edge, and the device was encapsulated with a cover glass slide and an appropriate epoxy. $J-V$ and $L-V$ measurements were performed with a semiconductor characterization system (Keithley 4200), a switching matrix (Keithley 7002), and a black test box mounted with a calibrated large-area Si-photodiode (18 $\times 18 \mathrm{~mm}^{2}$ ) for a semiautomated test. The voltage was swept from -5 to $5 \mathrm{~V}$ with a zero biased photodiode monitoring. Encapsulated PLED $L-V$ tests were performed at room temperature and in air. The EL emission spectra from PLEDs were evaluated using a spectrometer at room temperature under darkness by using a multimode optical fiber butt-coupled to a PLED pixel, while it was biased at about $5 \mathrm{~V}$. The active area of the devices was $0.19 \mathrm{~cm}^{2}$.

Figure 1 shows the forward-biased $J-V$ characteristics of the devices incorporated with $5 \mathrm{~nm}$ thick interlayers between the MEHPPV and Al. The control device with a plain Al cathode showed a current density of only $39 \mathrm{~mA} / \mathrm{cm}^{2}$ at $5 \mathrm{~V}$. An approximately three times larger current density was observed for the same bias when inserting a thin LiF interlayer between the MEH-PPV and Al. This cathodic Li-halide interlayer was then varied down the group VII column. Devices with a $\mathrm{LiBr}$ interlayer show even higher current densities than both the $\mathrm{LiF}$ and the $\mathrm{LiCl}$ devices with a current density of $231 \mathrm{~mA} / \mathrm{cm}^{2}$ at $5 \mathrm{~V}$. From the $J-V$ curves, it is clear that as the cathode interlayer is varied down the group VII column of the periodic table, the current density increases due to the enhanced electron injection. Table I summarizes the $J-V$ characteristics of the devices, illustrating the current density at $5 \mathrm{~V}$.

Figure 2 shows the $L-V$ characteristics of the devices. Comparing the luminance at $5 \mathrm{~V}$, devices with $\mathrm{LiF}, \mathrm{LiCl}$, and $\mathrm{LiBr}$ interlayers 


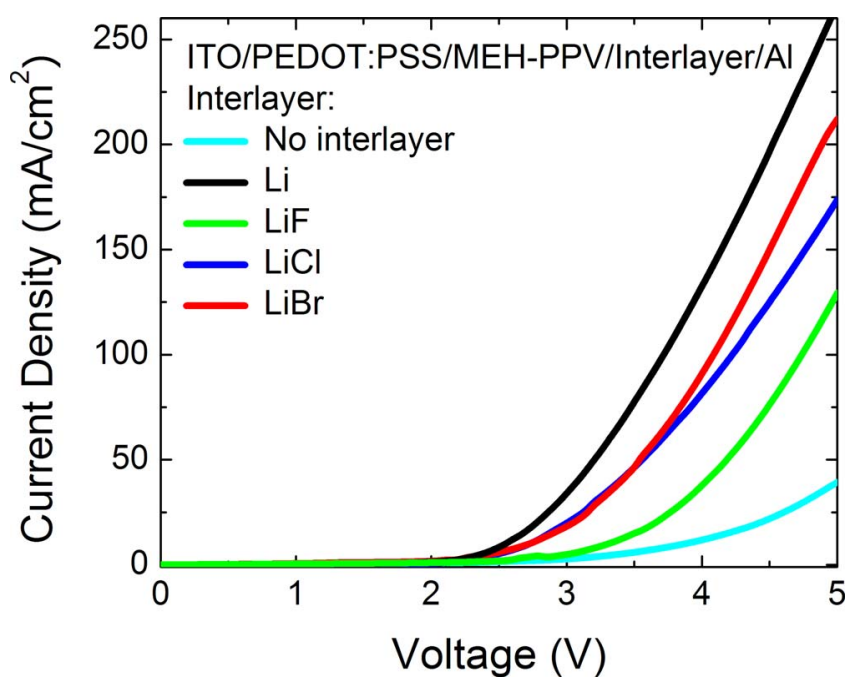

Figure 1. (Color online) $J-V$ characteristics of PLEDs with $5 \mathrm{~nm}$ thick Lihalide interlayers between the MEH-PPV and Al cathode (ITO/PEDOT:PSS/ MEH-PPV/Li-halide/Al). Two control devices were also tested, one without a Li-halide interlayer (ITO/PEDOT:PSS/MEH-PPV/Al) and the other with metallic Li (ITO/PEDOT:PSS/MEH-PPV/Li/Al).

show 583,1363 , and $1632 \mathrm{~cd} / \mathrm{m}^{2}$, respectively. The luminance from the device with a $\mathrm{LiBr}$ interlayer at a $5 \mathrm{~V}$ bias is slightly smaller than the luminance of the device with Li-only interlayer, $1609 \mathrm{~cd} / \mathrm{m}^{2}$ at $5 \mathrm{~V}$ (Table I). The observed modest luminance of the $\mathrm{Li} / \mathrm{Al}$ cathode devices can be interpreted as a greatly increased $\mathrm{Li}$ doping density penetrating deep into the MEH-PPV active layer and quenching luminescence efficiency. Considering the relation between the doping level and the depth of the doped region, increased Li doping into the MEH-PPV layer could lead to enhanced electron injection due to a low work-function contact at the interface, but meanwhile Li dopants can form quenching sites due to the longrange $\mathrm{Li}$ diffusion into the emission zone of MEH-PPV. ${ }^{11}$ As the Li-halide interlayers between the MEH-PPV and $\mathrm{Al}$ are varied down the group VII column, a reduced turn-on voltage is also observed. Table I summarizes the $L-V$ characteristics of the devices, illustrating the luminance at $5 \mathrm{~V}$ and the turn-on voltage.

Figure 3 shows normalized EL emission spectra of the devices at $5 \mathrm{~V}$. The emission peak occurred at around $2.1 \mathrm{eV}$ for all devices, independent of the interlayer and the current density. From the extracted full width at half-maximum (fwhm), the device with Al cathode only shows about $242 \mathrm{meV}$ of fwhm of the spectra, which is slightly narrower than that of the device with $\mathrm{Li} / \mathrm{Al}$ cathode $(252 \mathrm{meV})$. For the devices with $\mathrm{LiF}, \mathrm{LiCl}$, and $\mathrm{LiBr}$ interlayers, each extracted fwhm was 245, 250, and $279 \mathrm{meV}$, respectively. As

Table I. The current density $(J)$ at $5 \mathrm{~V}$, the luminance $(L)$ at $5 \mathrm{~V}$, and the turn-on voltage $\left(V_{\text {turn-on }}\right)$, defined at which $L$ $=0.2 \mathrm{~cd} / \mathrm{m}^{2}$, are extracted from the $J-V$ and $L-V$ characteristics of PLEDs (ITO/PEDOT:PSS/MEH-PPV/Li-halide/Al) with various $5 \mathrm{~nm}$ nominally thick Li-halides and two control devices (ITO/PEDOT:PSS/MEH-PPV/AI and ITO/PEDOT:PSS/MEH-

PPV/Li/AI). Results from the best devices are presented.

\begin{tabular}{lcccccc} 
& \multicolumn{2}{c}{ Control device cathode $^{\mathrm{a}}$} & & \multicolumn{3}{c}{ Li-halide cathode $^{\mathrm{a}}$} \\
\cline { 2 - 3 } \cline { 6 - 7 } & $\mathrm{Al}$ & $\mathrm{Li} / \mathrm{Al}$ & & $\mathrm{LiF} / \mathrm{Al}$ & $\mathrm{LiCl} / \mathrm{Al}$ & $\mathrm{LiBr} / \mathrm{Al}$ \\
\hline$J\left(\mathrm{~mA} / \mathrm{cm}^{2}\right)$ at $5 \mathrm{~V}$ & 39 & 291 & & 129 & 173 & 231 \\
$L\left(\mathrm{~cd} / \mathrm{m}^{2}\right)$ at $5 \mathrm{~V}$ & 0.9 & 1609 & & 583 & 1363 & 1632 \\
$V_{\text {turn-on }}(\mathrm{V})$ & 4.6 & 2.6 & & 3.6 & 3.4 & 3.2
\end{tabular}

${ }^{a}$ Device structure: ITO/PEDOT:PSS/MEH-PPV/Cathode.

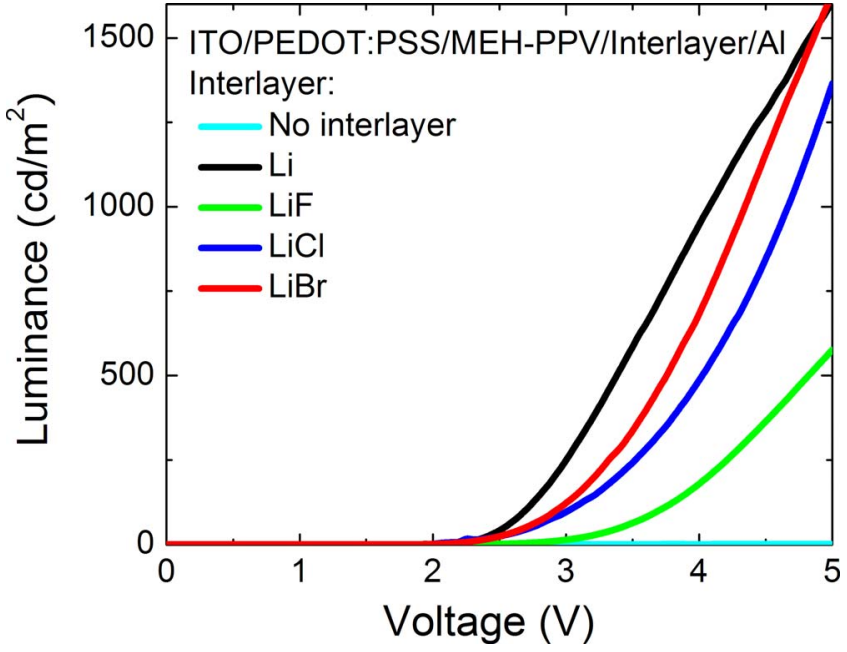

Figure 2. (Color online) $L-V$ characteristics of PLEDs with $5 \mathrm{~nm}$ thick Li-halide interlayers between the MEH-PPV and Al cathode (ITO/ PEDOT:PSS/MEH-PPV/Li-halide/Al). Two control devices were also tested, one without a Li-halide interlayer (ITO/PEDOT:PSS/MEH-PPV/Al) and the other with metallic Li (ITO/PEDOT:PSS/MEH-PPV/Li/Al).

Li-halide interlayers are varied down the group VII column, a small broadening of the EL emission spectra was observed, which might indicate an increase in electronic disorder and possible interfacialchemical activity between MEH-PPV and the Li-halide interlayer. Similar effects have been reported in the literature. ${ }^{12}$

Among the various mechanisms used to explain enhanced electron injection with thin metal-halide interlayers between the electroactive polymer and metal cathode, it is possible that the metal-halide salts dissociate and the Li ions diffuse into the EL polymer layer, where they donate electrons to the $\pi$-system, thereby increasing the electron density near the electron injecting contact, or by lowering the electron injection barrier in the polymer/cathode interface by introducing gap states in the energy gap of the bulk polymer semiconductor. $^{3}$ Thus, enhanced electron injection is observed, which also leads to higher EL external quantum efficiencies. Baldo

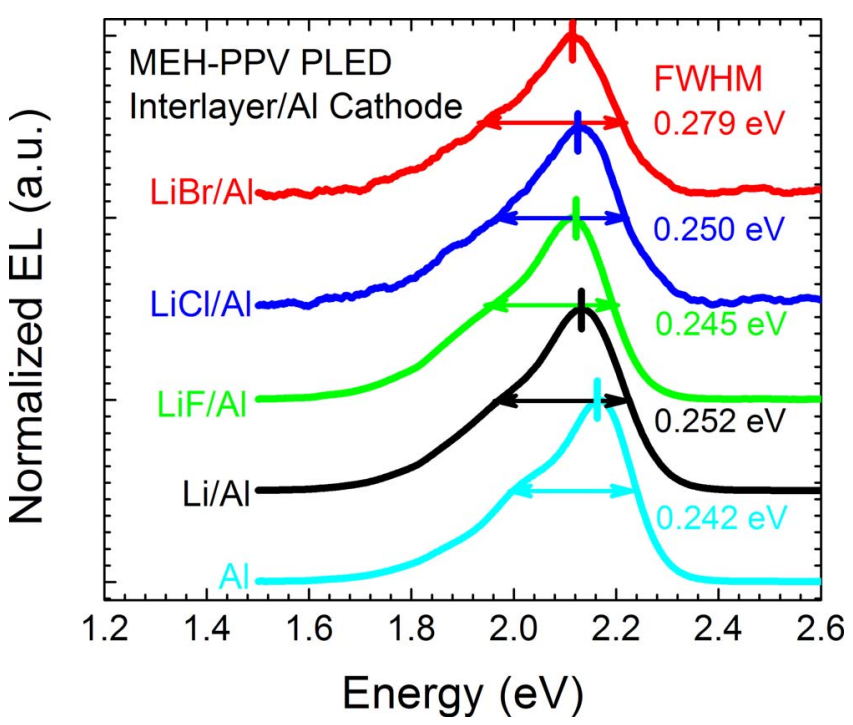

Figure 3. (Color online) The normalized EL spectra of PLEDs (ITO/ PEDOT:PSS/MEH-PPV/Li-halide/Al) with $5 \mathrm{~nm}$ thick various Li-halide interlayer and two control devices at the drive voltage of $5 \mathrm{~V}$. fwhm is extracted: Al (242 meV), Li/Al (252 meV), LiF/Al (245 meV), LiCl/Al (250 meV), and $\mathrm{LiBr} / \mathrm{Al}(279 \mathrm{meV})$. The plots have been shifted vertically. 
et al. reported that a thermally evaporated surface layer of metallic $\mathrm{Li}$ diffuses through, and subsequently dopes, the electrontransporting organic semiconducting thin film immediately below the cathode, forming an ohmic contact as evidenced by currentvoltage and secondary ion mass spectrometry data. ${ }^{13}$ Furthermore, photoemission spectroscopy supports the claim that enhanced electron injection by Li metal doping occurs, suggesting that Li metal lowers the barrier to electron injection at the polymer/cathode interface by introducing gap states in the forbidden gap of the polymer semiconductor. ${ }^{13,14}$ Similar behavior was also observed in the case of $\mathrm{Cs}$ dissociating from $\mathrm{CsF}$ upon deposition of $\mathrm{Al}$ and diffusing into and doping the underlying polymer layer. ${ }^{15}$

Generally, the lattice energy of ionic solids can be defined as the energy per ion required to separate completely into ions in a crystal lattice at a temperature of absolute zero. ${ }^{16}$ There is a possible correlation between the lattice energy of the Li-halide compound and the current density. $\mathrm{LiF}$ has the highest lattice energy of $1030 \mathrm{~kJ} / \mathrm{mol}$ and has the lowest device performance, followed in order of decreasing lattice energy $(834 \mathrm{~kJ} / \mathrm{mol})$ by the $\mathrm{LiCl}$ devices. Finally, the $\mathrm{LiBr}$ devices have the lowest lattice energy $(788 \mathrm{~kJ} / \mathrm{mol})$, concurrently with the highest current density. ${ }^{17}$ This result is possibly explained by the fact that Li-halide salts decompose, facilitating the diffusion of $\mathrm{Li}$ ions into the EL polymer. The electron mobility of the MEH-PPV is reported to be about 3 orders of magnitude lower than the hole mobility. ${ }^{18}$ In MEH-PPV PLED with various Li-halides interlayers, hole current dominates the current flow and the electrical properties of the devices are essentially the same as space-charge-limited hole-only devices. For such low electron mobility of the MEH-PPV, it is reported that the electron density can only be significant near the electron-injecting contact due to the low mobility of electron in the MEH-PPV. ${ }^{19}$ The resulting enhanced current densities, reduced turn-on voltages, and subsequent improved light output, as the lattice energy of the Li-halide compound decreases, could be due to a high surface charge density with increased $\mathrm{Li}$-ion doping density at the cathode interface in the EL layers of PLEDs. It is well known that alkali metals, such as Li, $\mathrm{Na}$, and $\mathrm{Cs}$, are used as efficient donors, and often they are introduced through the decomposition of materials like metal halides. However, the alkali cation is mobile and can also diffuse through the polymer layer. ${ }^{20,21}$ Zhao et al. reported improved PLED performance by doping the EL polymer layer with $\mathrm{LiBr}$, suggesting that doping with $\mathrm{LiBr}$ facilitates lowering charge-injection barriers at one or both electrode-polymer interfaces. ${ }^{22}$ A possible explanation is that the metal halides with higher atomic mass and radius are less diffusive and therefore create a greater concentrated effect at the hybrid interface, promoting better electron injection.

In this study, the PLED with a $\mathrm{LiBr}$ interlayer shows a similar performance to the PLED with a Li interlayer. However, the amount of free $\mathrm{Li}$ metal dissociated from the $\mathrm{LiBr}$ interlayer should be significantly less than the pure $\mathrm{Li}$ metal interlayer due to the lattice energy of $\mathrm{LiBr}$ compound and the creation of $\mathrm{Li}$ ions instead. Therefore, it is unlikely that Li-halide's dissociation-induced doping could be the only mechanism for the enhanced electron injection upon inserting Li-halides. As the lattice energy decreases, the dipole moment also increases for the Li-halide compounds as follows: $\mathrm{LiF}$ (6.3D), $\mathrm{LiCl}$ (7.1D), and $\mathrm{LiBr}(7.3 \mathrm{D}) .{ }^{23}$ An alternative mechanism, which could account for the increase in both $J$ and $L$ upon insertion of Li-halides, could be that an interfacial dipole is formed by the Li-halides at the interface. ${ }^{24}$ The strong interfacial dipole of $\mathrm{Li}$ halides at the interface could promote better band banding and enhanced electron injection.

In conclusion, the effects of Li-halides on electron injection were examined for EL layers of PLEDs by monitoring their $J-V$ and $L-V$. An order of magnitude increase in the current density was observed as halides were varied down the group VII column of the periodic table. When considering the light-output intensity, $\mathrm{LiCl}$ was $2.3 \times$ greater than $\mathrm{LiF}$, whereas $\mathrm{LiBr}$ was $2.8 \times$ greater, while lowering the turn-on voltage. This resulting enhanced current density, and subsequent luminance, could be due to a low work-function contact created by Li-halide dissociation-induced doping or an interfacial dipole of Li-halides at the interface as the lattice energy of the Lihalide compound decreased, leading to band bending.

\section{Acknowledgments}

This work was partially supported by the NSF (DMR-0103248).

The Ohio State University assisted in meeting the publication costs of this article.

\section{References}

1. T. M. Brown and F. Cacialli, J. Polym. Sci., Part B: Polym. Phys., 41, 2649 (2003)

2. J. H. Yoon, J. J. Kim, T. W. Lee, and O. O. Park, Appl. Phys. Lett., 76, 2152 (2000).

3. T. M. Brown, R. H. Friend, I. S. Millard, D. C. Lacey, J. H. Burroughness, and F. Cacialli, Appl. Phys. Lett., 79, 174 (2001).

4. X. Yang, Y. Mo, W. Yang, G. Yu, and Y. Cao, Appl. Phys. Lett., 79, 563 (2001)

5. L. S. Hung, C. W. Tang, and M. G. Mason, Appl. Phys. Lett., 70, 152 (1997).

6. M. A. Baldo and S. R. Forrest, Phys. Rev. B, 64, 085201 (2001).

7. J. Kido and T. Matsumoto, Appl. Phys. Lett., 73, 2866 (1998).

8. X. Y. Deng, S. W. Tong, and L. S. Hung, Appl. Phys. Lett., 82, 3104 (2003).

9. S. E. Shaheen, G. E. Jabbour, M. M. Morrell, Y. Kawabe, B. Kippelen, N Peyghambarian, M.-F. Nabor, R. Schlaf, E. A. Mash, and N. R. Armstrong, J. Appl. Phys., 84, 2324 (1998).

10. K. H. Lee, H. W. Jang, K. B. Kim, Y. H. Tak, and J. L. Lee, J. Appl. Phys., 95, 586 (2004).

11. Y. Cao, G. Yu, I. D. Parker, and A. J. Heeger, J. Appl. Phys., 88, 3618 (2000).

12. Y. Park, V. E. Choong, B. R. Hsieh, C. W. Tang, and Y. Gao, Phys. Rev. Lett., 78, 3955 (1997).

13. G. Parthasarathy, C. Shen, A. Kahn, and S. R. Forrest, J. Appl. Phys., 89, 4986 (2001).

14. M. G. Mason, C. W. Tang, L.-S. Hung, P. Raychaudhuri, J. Madathil, and D. J. Giesen, J. Appl. Phys., 89, 2756 (2001).

15. P. Piromreun, H. Oh, Y. Shen, G. G. Malliaras, J. C. Scott, and P. J. Brock, Appl. Phys. Lett., 77, 2403 (2000)

16. CRC Handbook of Chemistry and Physics, David R. Lide, Editor, Internet Version 2007, 87th ed., Taylor and Francis, Boca Raton, FL (2007) (Internet Version 2007, http:/www.hbcpnetbase.com).

17. CRC Handbook of Chemistry and Physics, 87th ed., David R. Lide, Editor, Taylor and Francis, Boca Raton, FL (2007) (Internet Version 2007, http:/ www.hbcpnetbase.com).

18. B. K. Crone, I. H. Campell, P. S. Davids, and D. L. Smith, Appl. Phys. Lett., 73 3162 (1998).

19. I. H. Campbell, D. L. Smith, C. J. Neef, and J. P. Ferraris, Appl. Phys. Lett., 75, 841 (1999).

20. Q. Pei, Y. Yang, G. Yu, C. Zhang, and A. J. Heeger, J. Am. Chem. Soc., 118, 3922 (1996).

21. S. J. Kang, D. S. Park, S. Y. Kim, C. N. Whang, K. Jeong, and S. Im, Appl. Phys. Lett., 81, 2581 (2002)

22. W. Zhao and J. M. White, Appl. Phys, Lett, 87, 103503 (2005).

23. R. Winkler and S. T. Pantelides, J. Chem. Phys., 106, 7714 (1997)

24. S. K. M. Jönsson, E. Carlegrim, F. Zhang, W. R. Salaneck, and M. Fahlman, J. Opt. Soc. Am. B, 44, $3695(2005)$ 